\title{
OPTICALLY THICK WINDS IN NOVAE
}

\author{
GIORA SHAVIV \\ Department of Physics, Asher Space Research Institute, and Miverva Center for \\ Non-linear Physics, Israel Institute of Technology, Haifa, Israel, 32000
}

\begin{abstract}
We discuss the theory of optically thick winds from Novae, how they are treated numerically, and the effect of the mass loss on the long term evolution of the WD.
\end{abstract}

Key words: stars: novae - white dwarfs - winds

\section{Introduction}

Novae are stellar episodes in which a mass shell of the order of $10^{-5} M_{\odot}$ is ejected as a consequence of a thermonuclear runaway (TNR) on White Dwarfs. The TNR is a consequence of accretion of hydrogen rich matter onto a cold WD. The accreted matter becomes degenerate and the hydrogen nuclear reactions start in a degenerate layer. Consequently, the temperature rises to $(1.5-4) \times 10^{8} \mathrm{~K}$ and radiation pressure drives the material to slightly supersonic velocities and induces mass loss from the system. Once almost the entire accreted mass is ejected from the star, the star cools, the accretion process eventually resumes and the cycle starts again. The phenomenon is therefore, periodic (as long as the secondary pours its mass on the primary). The analysis of the nova ejecta shows that there are two kinds of WD, (a) composed of mainly $\mathrm{C} / \mathrm{O}$ and (b) composed mainly of $\mathrm{O} / \mathrm{Ne} / \mathrm{Mg}$.

The energy release by hydrogen burning (via the CNO or the $\mathrm{NeNaMg}$ cycles) is marginally sufficient to eject the envelope off the WD. This can be deduced from the observational fact that the velocities of the ejecta are smaller than the escape velocities from the surface of the WD. The expansion of the envelope to very large radii and the reduction in the escape velocity is critical to the mass loss in a form of an optically thick wind. The TNR phenomenon is not sufficiently violent to create shocks and subsequently eject the envelope in a 'single outburst'. This is the reason why we see a continuous mass loss via a wind. Important roles in the ejection energy input are played by the spread of the $\beta$-unstable nuclei in the expanding envelope and the motion of the secondary inside the envelope since in most cases the nuclear energy released is not sufficient to eject the envelope. Consequently, for low $\dot{m} s$ and almost irrespective of the mass of the WD one finds mass ejection. However, at high $\dot{m} s$ and high masses the accreted matter may not become sufficiently degenerate and the following TNR is relatively mild with the consequence that the mass ejected, if any, is less than the accreted mass leading to an increase in the mass of the WD. Thus the picture of accretion induced collapse (AIC) is rather complicated because the mass of the WD is also a parameter and not only $\dot{m}$. The details of the mass ejection 
mechanism and its numerical treatment are important for the question of the long term evolution of the WD.

Several mechanisms act to provide the energy to the wind, among them radiation pressure and heating by radioactive nuclei decaying in the expanding envelope. We discuss here only the effect of the radiation pressure since its effect on the algorithm for mass loss is crucial.

The discussion here is restricted to the physics of the mass loss, how the mass loss occurs and how it is treated in the evolutionary calculations. The question how it is observed, namely the spectra of expanding envelopes, is a very interesting topic but time does not permit a proper discussion. Several additional mechanisms instrumental in the mass loss, like expansion opacity, will not be covered either.

\section{Treating the mass loss}

The first treatment of an optically thick wind was by Bath and Shaviv (1976, BS). BS assumed a steady state wind and derived various consequences. BS did not consider the causes, namely the mechanisms of the wind. On the other hand, hydrodynamic simulations have the problem of treating a wind along with the internal structure in a consistent way. Prialnik et al. (1982) were the first to have implemented several assumptions, like supersonic flow velocities greater than the local speed of sound, velocities greater then the escape velocity etc. to handle continuous mass loss in evolutionary calculations. The theory of mass loss presented here was worked out in collaboration with $\mathrm{A}$. Kovetz and is the latest version used in stellar hydrodynamics with continuous optically thick mass outflow. In view of the far reaching consequences to the theory of accretion onto compact objects and the theory SN Type I etc. the discussion will be slightly detailed.

We begin by deriving the wind equation under the condition of optically thick expanding envelope. In a steady flow, balance of (radial) momentum requires

$$
\frac{\partial v}{\partial t}+v \frac{d v}{d r}=-\frac{1}{\rho} \frac{d P}{d r}-\frac{G m}{r^{2}}
$$

If we separate the pressure into gas pressure $P_{g}$ and radiation pressure $P_{r}$, we obtain

$$
d P=d\left(P_{g}+P_{r}\right)=\frac{\partial P_{g}}{\partial \rho} d \rho+\left(1+\frac{\partial P_{g} / \partial \log T}{4 P_{r}}\right) d P_{r} .
$$

We introduce the notation $c_{s}^{2}=\partial P_{g} / \partial \rho$ for the squared (isothermal) speed of sound and note that $1+\left(\partial P_{g} / \partial \log T\right) / 4 P_{r}=(\partial P / \partial \log T) / 4 P_{r}$.

Since the wind is optically thick we can use the diffusion approximation for the radiative flux and write

$$
L_{r}=-4 \pi r^{2} \frac{c}{\kappa \rho} \frac{d P_{r}}{d r}
$$


for the radiative luminosity. No relativistic corrections are needed in this case. The mass flow is given by

$$
\dot{m}=4 \pi r^{2} \rho v,
$$

we can write (1) in the form

$$
\frac{\partial v}{\partial t}+\frac{c_{s}^{2}}{\dot{m}} \frac{\partial \dot{m}}{\partial r}+\left(v^{2}-c_{s}^{2}\right) \frac{1}{v} \frac{d v}{d r}=\frac{2 c_{s}^{2}}{r}-\left(1-\frac{\partial P / \partial \log T}{4 P_{r}} \frac{L_{r}}{\mathrm{~L}_{E d}}\right) \frac{G m}{r^{2}} .
$$

In (6), $\mathrm{L}_{E d}$ is the Eddington luminosity, defined by $\mathrm{L}_{E d}=4 \pi c G m / \kappa$.

In steady state and when the mass loss does not depend on $r$, we get that at the sonic point, where $v=c_{s}$, the left hand side of (6) vanishes, and so must the right hand side. Thus, at the sonic point,

$$
\frac{L_{r}}{\mathrm{~L}_{E d}}=\frac{1-2 c_{s}^{2} r / G m}{1+\left(\partial P_{g} / \partial \log T\right) /\left(4 P_{r}\right)}=\frac{1-4\left(c_{s} / v_{e s}\right)^{2}}{1+\left(\partial P_{g} / \partial \log T\right) /\left(4 P_{r}\right)} .
$$

The last results implies that in a steady state the luminosity never exceeds the Eddington luminosity in subsonic regions. Further out, however, $L_{r}$ may exceed $\mathrm{L}_{E d}$.

In a nova outburst, heating by nuclear reactions may cause the star to expand to giant dimensions. The material may (asymptotically) attain a positive, 'final' outward velocity $v_{\infty}$. A standard Lagrangian evolution code cannot follow the details of this process: quite apart from the practically infinite radii, the relation (4) must fail as soon as the optical depth becomes of the order of unity. The obvious procedure, once the star has expanded, is to detect mass loss and to subtract mass from the star at the rate $\dot{m}$. The details of this prescription are very important to questions like when it happens, what is the effective temperature as well as how far does the mass loss go, namely is the entire hydrogen rich layer removed or is there some hydrogen left, or does the burning converts significant amounts of hydrogen into helium and does the helium remain on the star?

We shall now derive a formula for $\dot{m}$ which is appropriate under the conditions that prevail in expanding nova envelopes. Specifically, we shall assume that, at $r=r_{0}$, close to the stellar photosphere,

$$
v\left(r_{0}\right) \gg c_{s}\left(r_{0}\right), \quad 2 c_{s}^{2}\left(r_{0}\right) \ll \frac{G M}{r_{0}}=1 / 2 v_{e s c}^{2}\left(r_{0}\right) .
$$

The first condition is marginally satisfied in novae. In what follows, we shall use the abbreviated notations

$$
f=\left(1-\frac{L}{\mathrm{~L}_{E d}}\right)=1-\frac{\partial P / \partial \log T}{4 P_{r}} \frac{L_{r}}{\mathrm{~L}_{E d}},
$$




$$
V_{0}^{2}=\left(1-\frac{L}{\mathrm{~L}_{E d}}\right) \frac{2 G m}{r_{0}}=f v_{e s c}^{2} .
$$

We recall that, in accordance with the foregoing remarks, $V_{0}^{2}$ may (despite the notation) become negative however, we shall not take the square root of $V_{0}^{2}$. Under the assumptions (8) and steady state and constant (in space) mass loss, we can replace (6) (for $r \geq r_{0}$ ) by the differential equation

$$
v d v=-\left(1-\frac{L}{\mathrm{~L}_{E d}}\right) \frac{G m}{r^{2}} d r .
$$

If, in addition to the assumptions (8),

$$
v^{2}\left(r_{0}\right) \geq V_{0}^{2}=f v_{e s c}^{2},
$$

equation (11) will have the solution

$$
v^{2}(r)=v^{2}\left(r_{0}\right)-V_{0}^{2}\left(1-r_{0} / r\right),
$$

and the material will attain the final speed $v_{\infty}$ given by

$$
v_{\infty}^{2}=v^{2}\left(r_{0}\right)-V_{0}^{2}>0 .
$$

We note that (12) will be identically satisfied whenever $V_{0}^{2}<0$, that is, whenever $L>\mathrm{L}_{E d}$ (and $f<0$ ). The quantity $f$, defined in equation (9) is the effect of the radiation pressure and was hitherto never taken into account.

The optical depth (from $r_{0}$ to infinity) is

$$
\tau_{0}=\int_{r_{0}}^{\infty} \kappa \rho d r=\frac{1}{4 \pi} \int_{r_{0}}^{\infty} \frac{\dot{m} \kappa d r}{r^{2} v} .
$$

We take an average value of $\kappa \dot{m}$ outside the integral and use (11):

$$
\begin{aligned}
& \tau_{0}=-\frac{\langle\kappa \dot{m}\rangle}{4 \pi} \int_{r_{0}}^{\infty} \frac{d r}{r^{2} v}=-\frac{\langle\kappa \dot{m}\rangle}{4 \pi} \int_{v_{0}}^{v_{\infty}} \frac{d r}{\left(1-L / L_{E d}\right) G M} \\
& =-\frac{\langle\kappa \dot{m}\rangle}{4 \pi r_{0}} \frac{2}{\left\langle V_{0}^{2}\right\rangle}\left(v_{\infty}-v\left(r_{0}\right)\right)=-\frac{\langle\kappa \dot{m}\rangle}{4 \pi r_{0}} \frac{2}{\left\langle V_{0}^{2}\right\rangle} \frac{v_{\infty}^{2}-v_{0}^{2}}{\left(v_{\infty}+v_{0}\right)} .
\end{aligned}
$$

Thus we have the following formula for the average mass loss rate:

$$
\langle\dot{m}\rangle=\tau_{0} \frac{4 \pi r_{0}}{\langle\kappa\rangle} \frac{v_{\infty}+v\left(r_{0}\right)}{2 g},
$$

where $g=\langle\kappa \dot{m}\rangle /\langle\kappa\rangle\langle\dot{m}\rangle \approx 1$. In this equation, we must have $\tau_{0} \geq \tau_{p h} \approx 2 / 3$ if the radiative diffusion formula (4) is to be valid. In accordance with (10) 
and (13), $\dot{m}$ will increase with $r_{0}$ (for given $\kappa, \tau_{0}$ and $v\left(r_{0}\right)$ ), roughly as $\sqrt{r_{0}}$. The formula (16) can also be written in the alternative form

$$
\dot{m}=\tau_{0} L_{E d} \frac{v_{\infty}+v\left(r_{0}\right)}{c v_{e s}^{2}},
$$

where $c$ is the speed of light and $v_{e s}^{2}=2 G M / r_{0}$ and $\mathrm{L}_{E d}$ is defined with $\langle\kappa\rangle$. Thus $\dot{m}$ is of order $\tau_{0} \mathrm{~L}_{E d} / c v_{e s}$. The rate at which kinetic energy is carried off by the wind is of order $\dot{m} \frac{1}{2} v_{e s}^{2} \approx \mathrm{L}_{E d} v_{e s} / c$.

Another form in which we can write (16) involves the mass $\Delta m$ lying above $r_{0}$. Using the relation $\tau_{0}=\langle\kappa\rangle \Delta m / 4 \pi r_{0}^{2}$, equation (16) becomes

$$
\dot{m}=\Delta m \frac{v_{\infty}+v\left(r_{0}\right)}{2 r_{0}} .
$$

Thus $\Delta m$ will be lost after a time $\tau_{\text {loss }}=2 r_{0} /\left(v_{\infty}+v\left(r_{0}\right)\right)$. For $r_{0}=R_{w d}$ $r_{0} / v_{e s} \approx 1$ second. The mass loss, as a rule, takes place when $r \cong 100 R_{w d}$ and hence the typical time scale is 100 seconds. In a giant, $r_{0} / v_{e s}$ is $\approx 1$ day. These time estimates assume that $v\left(r_{0}\right) \ll v_{e s}$ : if $v\left(r_{0}\right)$ is large, the time estimates are reduced by a factor $v_{e s} / v\left(r_{0}\right)$. Equation (18) is formally independent of $\kappa$ (or $\tau_{0}$ ), but it only holds when $\tau_{0} \geq \tau_{p h}$ (and when the assumptions (8) and (12) are satisfied).

In addition to the momentum balance wind equation (6), we must also consider the energy balance namely,

$$
\dot{m} d\left(u+\frac{P}{\rho}+\frac{1}{2} v^{2}-\frac{G M}{r}\right)+d L=0,
$$

where, as in (11), the differentials imply space derivatives. If we neglect the enthalpy $u+P / \rho$, which is essentially the second assumption of $(8)$, and use (13), equation (19) determines the change in $L$ throughout the region above $r_{0}:|\Delta L|=\dot{m}\left(L / \mathrm{L}_{E d}\right)\left(G M / r_{0}\right)$. According to the mass loss rate formula (16), this gives

$$
\frac{\Delta L}{L}=\tau_{0} \frac{v_{\infty}+v\left(r_{0}\right)}{2 c},
$$

which is small, as long as $\tau_{0}$ is not too large. This is a consistency check of the use of a constant (uniform) $L$ in the derivation of (16) and implies that the criteria for mass loss should not be applied at a too deep layer into the expanding star.

\section{The conditions for mass loss and some typical results}

We are now in a position to assess the conditions for mass loss from the last mass shell. The conditions are eq.(8) and $\tau_{0} \approx 1$. Thus the velocity must be supersonic and the gas temperature must be well below the virial one. 
Fig. 1. The bolometric luminosity as a function of time for an $\dot{m}=10^{-9} \mathrm{M}_{\odot} /$ yr on a 1.07 $\mathrm{M}_{\odot}$ WD.
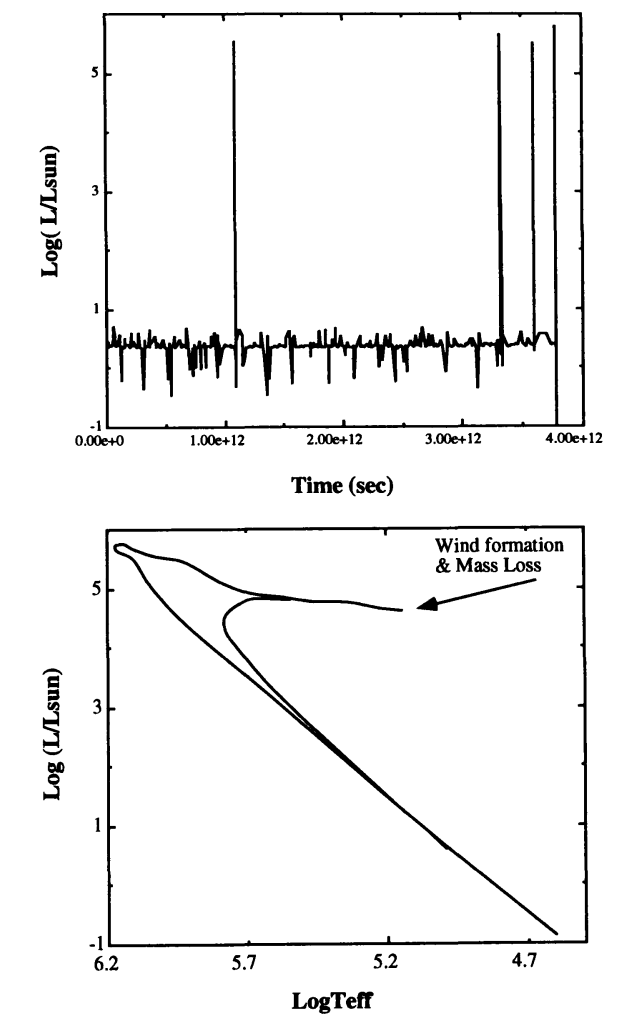

Fig. 2. The L-Te diagram for an erupting Nova. The mass of the WD is $1.07 \mathrm{M}_{\odot}$ and $\dot{m}=$ $10^{-9} \mathrm{M}_{\odot} / \mathrm{yr}$.

No hot gas outflow is possible
always in the last mass shell.

In the examples to be shown the initial model is a WD with a low central temperature ( in the range $8-30 \times 10^{6} \mathrm{~K}$. The masses of the WD in the calculations presented here are $1.07 \mathrm{M}_{\odot}$ and $1.40 \mathrm{M}_{\odot}$ with a composition of $50 \% C^{12}$ and $50 \% O^{16}$. The temperature of the core is $15 \times 10^{6} \mathrm{~K}$.

Figure 1 shows the long time evolution of a typical WD for $\dot{m}$ that leads to a CV. The luminosity is quite constant for a long time and then rises very quickly. The rise is the TNR. Note that the time between the first flashes is not identical to the time between the later ones. The reason is the sensitivity of the first flashes to the initial conditions which are unknown. Hence it is calculate several flashes if a result independent of the initial conditions is sought. Figure 2 shows the L-Te diagram where the mass loss starts via an optically thick wind. The tracks of two eruptions are shown. The surge to very high luminosities appears only in the first flash, manifesting again the difference between the first flashed and the next ones. The photosphere is $f e w \times 10^{5} \mathrm{~K}$. In figure 3 we show the dependence of the mass loss rate and the ejection velocity on time for two typical episodes. The fluctuations in the velocity are due to the finite size of the last mass shell and the variation in 
Fig. 3. The mass loss rate and velocity as a function of time for two mass loss episodes.

Fig. 4. The rate of mass loss and the mass last shell during the mass loss period.

Fig. 5. The mass loss rate and the hydrogen fraction of the ejected material for successive flashes.
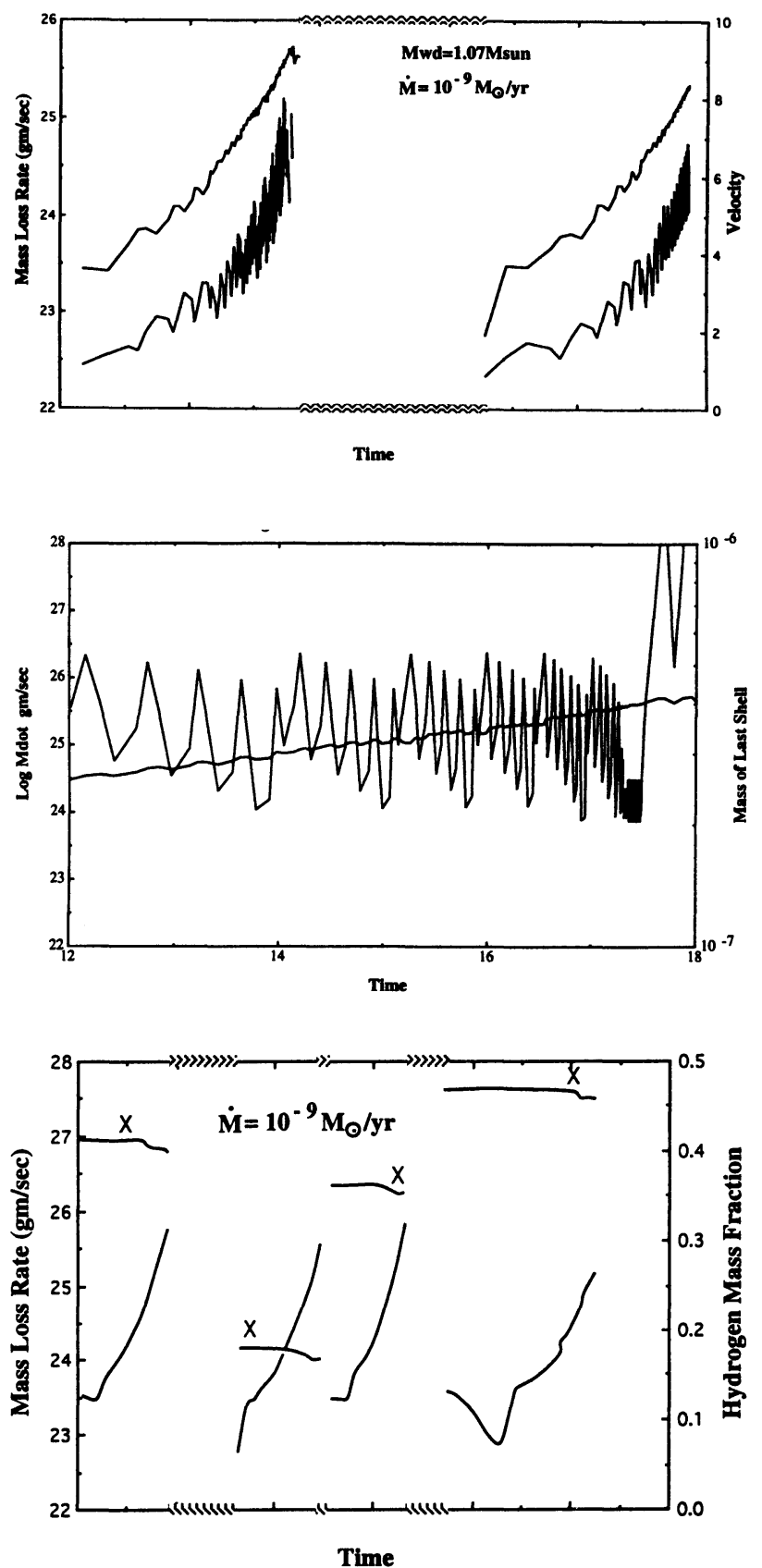

its size is due to the mass loss. Note the small variations in the instantaneous mass loss. A prerequisite for such a smooth behavior is a large number of properly sized mass shells (about 100 in this case). As a rule, the ejection velocity increases with time and with it the rate of mass loss. The details of 
the calculation are seen in figure 4 where the mass loss rate is shown along with the value of the last mass shell. The mass of the last mass shell oscillates because the process of mass removal does not remove an entire shell unless its mass is below a certain minimum. Each peak corresponds to a different mass shell and, as can be seen from the figure, many shells participate in the process. While the oscillations in the mass shells are rather large, the mean rate of mass loss changes slowly. The rate of mass loss changes by about a factor of $10^{2}$ during the mass loss episode.

The variation of the hydrogen mass fraction of the lost material during the different mass loss episodes is shown in figure 5 . The rate of the mass loss increases with time but the hydrogen mass fraction is almost constant except for the end of the mass loss process.

\section{When does an optically thick wind appear?}

The problem of accretion onto a cool WD has many free parameters: the temperature of the accreting WD, its mass and composition, the $\dot{m}$, the rate of WD illumination by the hot boundary layer, how fast does the accreted layer rotate? We discuss here what appears to be the most important ones, namely the mass of the WD and the $\dot{m}$. Clearly, accretion onto say a WD the composition of which is predominantly $\mathrm{Fe}$ will be very different from the present case where the constituents of the WD act as catalysts in the synthesis of hydrogen into helium. We consider two typical cases, one a high mass WD and a low mass WD.

A $1.4 \mathrm{M}_{\odot}$ C/O WD: We consider first the long term evolution of a $1.4 \mathrm{M}_{\odot}$ under various $\dot{m}$ s. The crucial points are: is there a wind with substantial mass loss and if yes how deep into the envelop the mass loss penetrates, in particular, is the entire envelop removed.

$\dot{m}=10^{-6} \mathrm{M}_{\odot}$ : One finds very frequent flashes around the Eddington luminosity. After a long series of small flashes, the model settles onto a steady burning of hydrogen without any mass loss. While some mass loss may occur during the decay of the initial conditions, there is no mass loss in the steady state. A helium layer between the hydrogen rich matter and the core grows. The nuclear evolution is fast and the helium layer is built before any diffusion of hydrogen into the core and of $\mathrm{C} / \mathrm{O}$ out of the core can take place. It is obviously not clear what will happen if shear mixing is the mechanism responsible for the enrichment of the envelope with heavy elements. $\dot{m}=10^{-7} \mathrm{M}_{\odot}$ : The model undergoes regular flashes which are sharper in time relative to those seen in the previous $\dot{m}$. Yet, the final outcome is the same, a layer of helium forms at the base. At the beginning one can see a modest effect of the diffusion but as the layer of helium grows the effect decreases.

$\dot{m}=10^{-8} \mathrm{M}_{\odot}$ : This $\dot{m}$ leads to successive flashes. The period between 
flashes is about $20 \mathrm{yr}$ and the amplitude of about $10^{3.5}$ in the bolometric luminosity. The TNR develops into a mass loss phase which ends with a complete removal of the newly accreted envelope. The WD is eroded and decreases in mass. The eroded mass is about $20 \%$ of the accreted one, which is about $2 \times 10^{-7} \mathrm{M}_{\odot}$. However, due to burning of matter which diffused into the core, the last layer on the WD is enriched with hydrogen. This hydrogen burns to helium during the time interval between the mass loss and the mass accretion phases. At the moment the next flash start the mass of the helium layer is about $2.5 \times 10^{-8} \mathrm{M}_{\odot}$ and it does not change in size with successive flashes.

$\dot{m} \leq 10^{-9} \mathrm{M}_{\odot}$ : This is the domain of the classical nova eruptions. Diffusion brings hydrogen to the core and the mass erosion of the WD is largest. The enrichment of the envelope with heavy elements is inversely proportional to the $\dot{m}$. This is the classical case of an optically thick wind. The typical rates of mass loss are $10^{23}-10^{25.6} \mathrm{gm} / \mathrm{s}$. The ejection velocities vary from 300 $\mathrm{km} \mathrm{s}^{-1}$ for the high $\dot{m}$ to $2500 \mathrm{~km} \mathrm{~s}^{-1}$ in the low $\dot{m}$ case.

A $1.07 \mathbf{M}_{\odot}$ C/O WD : For $\dot{m} \leq 10^{-6} \mathrm{M}_{\odot} M_{W D}$ decreases. However, for high $\dot{m}$ s one finds that the entire envelope is all the time convective and hence the helium formed at the base is continuously mixed in the envelope and no helium rich layer is formed at the end of the mass loss episode.

A $0.6 \mathrm{M}_{\odot} \mathrm{C} / \mathrm{O}$ WD : For $\dot{m} \leq 10^{-9} \mathrm{M}_{\odot}$ the $M_{W D}$ decreases.

\section{Acknowledgements}

Many years of collaborations and discussions with Attay Kovetz are immensely acknowledged. This research was supported by the VIP foundation at the Technion.

\section{References}

Bath, G.T., Shaviv, G. 1976, MNRAS 175, 305

Prialnik, D., Livio, M., Shaviv, G., Kovetz, A. 1982, ApJ 257, 312

Schwartzman, E., Kovetz, A., Prialnik, D. 1994, ApJ submitted 


\section{DISCUSSION:}

Cherepashchuk: Could you get such an anisotropy of wind without taking into account viscosity of matter?

Shaviv: This particular calculation was carried out with $\operatorname{Re}=10^{7}$ (Reynolds number). So very small viscosity. We do not know what happens at $\operatorname{Re} \sim 10^{14-16}$ and what viscosity we should use. Also, I showed a case without rotation.

Pols: At what rate does the mass of the white dwarf actually grow in your calculations, if the accretion rate is $10^{-6}$ or $10^{-7} \mathrm{M} \odot / \mathrm{yr}$ ? Considering that one needs a massive companion to produce such mass transfer rates, hence a short accretion timescale $\left(\leq 10^{6} \mathrm{yr}\right)$, does the WD accrete enough mass to actually evolve to AIC, except if $M_{W D}$ is very close to the Chandrasekker mass?

Shaviv: The rate of $M_{\text {wD }}$ growth is $10^{-8} \mathrm{M} \odot$ per cycle. The amount of mass growth varies. It is low at the beginning (since some mass is ejected) and then decreases.

You are right in stating that unless the WD is already very massive it will take a very long time for a $\mathrm{C} / \mathrm{O}$ WD to accrete hydrogen rich material and issue an AIC. However, if you accrete $\mathrm{He}$ (say pure helium) then $\mathrm{M}_{\mathrm{WD}}=\mathrm{m}_{\mathrm{acc}}(\mathrm{He})$ and you gain 3 orders of magnitude.

Koenigsberger: What is the timescale for the case of $\dot{\mathrm{M}} \sim 10^{-6} \mathrm{M}_{\mathrm{o}} / \mathrm{yr}$ for the $\mathrm{L}_{\text {bol }}$ and $\mathrm{T}$ variations? How would enhanced He abundances in the accreted matter affect the results?

Shaviv: The timescale for $\mathrm{L}_{\mathrm{Bo}}$ and maximum temperature variation is months, at this high accretion rate. If you change the abundance of the accreted material, say from hydrogen rich to helium rich, then their will be no mass loss (for any $\mathrm{M}_{\mathrm{acc}}$ ) since the energy output in the $3 \alpha$ reaction is so much smaller than the energy output in the $4 \mathrm{p} \rightarrow \mathrm{He}^{4}$ reaction. As a consequence, He acts like a poison to the thermonuclear runaway.

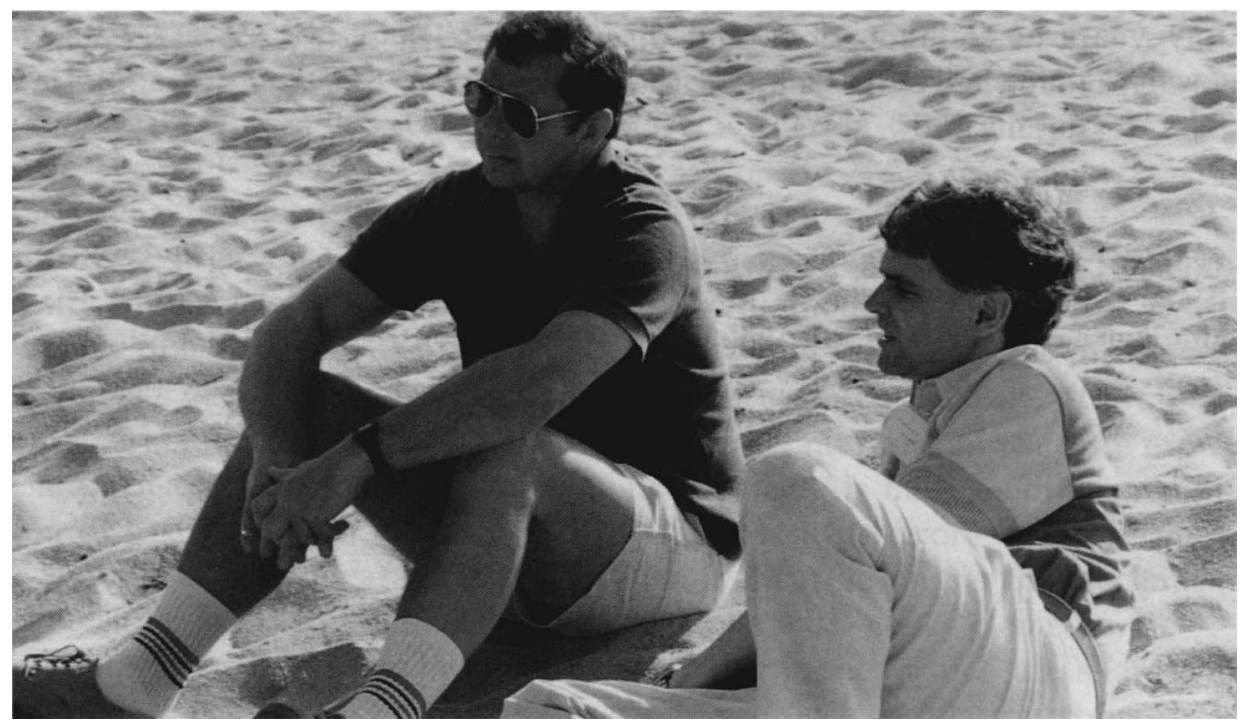

Stickland, Bode 